\title{
LES SPÉCIFICITÉS DU DISCOURS INDIRECT DANS L'ÉCHANGE ORAL SPONTANÉ EN SLOVÈNE
}

\section{INTRODUCTION}

Les analyses du discours rapporté, qu'il soit direct ou indirect, ont trait généralement à la langue écrite standard. Ainsi, dans La Grammaire du slovène (Slovenska slovnica, ouvrage de référence en matière de grammaire slovène), nous lisons :

Nous pouvons rapporter à un destinataire un acte de parole primaire /.../ de quatre façons différentes $/ . . . /$ a) tel quel, sans le modifier, donc sans phrase introductive /.../ : il s'agit de la reprise littérale $/ . . . /$ b) en citant littéralement ce qui a été dit tout en précisant, si besoin est, dans une phrase spéciale, dite introductive, qui parle ou pense et quand, où, comment, pourquoi (etc.) il le fait : c'est le discours direct /.../; c) nous rapportons ce qui a été dit sous la forme d'une phrase subordonnée complétant un verbe d'énonciation ou d'opinion inclus dans l'énoncé de la phrase introductive /.../ ; č) nous rapportons ce qui a été dit à la troisième personne sans avoir recours à une phrase introductive : c'est le discours indirect libre ou discours semi-direct. /.../ Le discours direct se caractérise tout particulièrement par la présence de phrases introductives où le rôle principal revient au verbe (ou autre expression) d'énonciation ou d'opinion, par exemple reči (dire), povedati (dire), obljubiti (promettre), vprašati (demander), misliti si (penser en soi-même), etc. Le verbe de ce type peut également être omis, laissant place dans la phrase introductive exclusivement à d'autres circonstances plus importantes (par exemple : Marjeta jo je slišala in spodbudila - la mention de l'énonciation z besedami (par ces mots) ou rekoč (en disant) est ommise) (Toporišič 2000 : 653-655).

Si nous nous concentrons sur le discours rapporté direct et sur la phrase qui l'introduit, nous constatons que Slovenska slovnica propose une définition selon laquelle la phrase introduisant le discours rapporté comprend toujours un verbe d'énonciation ou d'opinion, ou bien un autre verbe exprimant un état d'esprit : " Dans la phrase introductive, le verbe d'énonciation peut-être omis et remplacé par un verbe exprimant un étant d'esprit ou une réaction /.../ ( (Toporišič $2000: 656$ ).

Nous trouvons une définition semblable de la phrase introductive (ou accompagnatrice) chez Martina Križaj Ortar :

Dans le discours direct, comme dans le discours indirect, il s'agit d'exprimer par des mots l'acte de parole primaire, et ce en ayant le plus souvent recours à des verbes d'énonciation, à des participes passés ou présent formés à partir de verbes d'énonciation /.../. La transmis-

\footnotetext{
*Adresse de l'auteur : Filozofska fakulteta, Oddelek za slovenistiko, Aškerčeva 2, 1000 Ljubljana, Slovénie. Mel : mojca.smolej@ff.uni-lj.si
} 
sion à l'aide des verbes d'énonciation est primordiale ; ces derniers sont inclus dans l'énoncé de ce que l'on appelle la phrase accompagnatrice/introductive (Križaj Ortar 1997 : 160).

Dans le présent article, nous nous intéresserons avant tout à l'analyse des phrases introductives du discours rapporté, mettant ainsi en valeur les éléments que les composent dans la langue parlée. Notre hypothèse est que le discours rapporté et les éléments constituant les phrases introductives accompagnant le discours direct diffèrent suivant que nous les rencontrons dans la langue standard écrite ou dans la langue parlée. Nous concentrerons notre attention sur les caractéristiques du discours rapporté direct propres à la langue parlée.

\section{CARACTÉRISTIQUES DES ENREGISTREMENT FORMANT LE CORPUS}

Le corpus qui sert de base à notre analyse du discours rapporté comporte trente échantillons textuels de langue parlée produits de manière spontanée par trente locuteurs différents.

Pour permettre une analyse linguistique cohérente du point de vue de l'appartenance régionale ou dialectale, les locuteurs sélectionnés sont tous originaires de Ljubljana ou des environs. En revanche, ils diffèrent par leur sexe (10 hommes et 20 femmes), leur âge (entre 21 et 72 ans) et leur niveau d'instruction.

Seuls deux intervenants participaient à chaque discours : le locuteur et le récepteur. Du fait qu'aucune durée minimale ou maximale ne leur était imposée, les locuteurs ont produit des textes de longueurs différentes, enregistrés à l'aide d'un dictaphone. L'enregistrement de tous les discours a duré environ 15 heures (la longueur totale des textes est de 181483 signes espaces comprises).

Pour mettre par écrit le discours, nous avons eu recours à la transcription, qui favorise la compréhension et la lisibilité aux dépens de la précision des phonèmes. Nous avons donc utilisé la transcription orthographique pour établir les trente textes constituant le corpus. En dépit de ce principe de base, on rencontre quelques exceptions où ont été retranscrites des formes introuvables dans le dictionnaire de la langue standard. Nous avons opté pour la prise en compte de ces exceptions lorsque celles-ci sont communes à tous les locuteurs enregistrés et lorsqu'elles n'entravent pas la lecture.

\section{CATÉGORIES DE DISCOURS RAPPORTÉ DANS LA LANGUE PARLÉE}

Suite à notre analyse, nous remarquons également dans la langue parlée différentes catégories de discours rapporté que nous pouvons classer dans trois grands groupes :

a) Le groupe du discours rapporté direct avec phrase introductive. On y distingue cinq sousgroupes en fonction de la forme que revêt la phrase introductive :

Exemples:

- le vrai discours direct : Pol se mi je enkrat /.../, se mi je Andreja opravičvala /.../, je rekla: »Joj, ej, jaz sem ti pa takrat težila, ej, šit, ne, sori.« Jaz sem rekla: »No, saj zdaj vidiš, kako je to, ne.« (B1) 
- Le faux discours direct : No, po sem pa še tist, sem se udal v usodo, ne, sem reku, zdaj bo dal mir, k bo vidu, kaj je notr, kakšna stvar, ne, kaj za sestavt, al, ne vem, ne. (B30)

- Le vrai discours direct avec ellipse partielle du verbe d'énonciation/d'opinion dans la phrase introductive : In kar naprej sem: »A bosta že luč ugasnile?« (B5)

- Le vrai discours direct avec ellipse totale du verbe d'énonciation/d'opinion dans la phrase introductive : In pol, k je blo konc, on tist: »Ja, Maja, počakajte, počakajte.« (B2)

- Le vrai discours direct avec réalisation partielle de la phrase introductive ou introductive : Pride en, a je to stažist, al kaj jaz vem kaj /.../ mislim, da je stažist, ne, pa tud če ne, no: »Ja, bomo kar mehur prepiknil, ne.« (B1)

b) Le discours rapporté direct sans phrase introductive annonçant la reprise du discours primaire.

Exemples:

- Dons, ne, v knižnci, pride k pultu en fant /.../ pa prav, da bi rad Letino tehniko 2 /.../. Po pa sem rekla: »A mate to za slovenščino? « Prav: »Ja.« Sem rekla: »Najbrž knjigo Ninina pesnika 2.«»Ja, ja.«(B20)

- Ja, joj, ne, take majo, čaki, to je tud ena, k je pršla, dobr maš maš, ka pa jaz vem, $\{e m\}$ Antigono od Šekspira, al pa, čaki ena je enkrat rekla, da bi SHAKESPEARA, ne, pa to to se je eni drugi knižničarki zgodl, ne, pa prav, da bi od SHAKESPEARA, tko, »Ja, posluš, ne, ti si drug letnik, še..., že tok let maš angleščino, saj to bi lohk vedla, da se reče Šekspir pa to je tud splošna izobrazba.«»Ja, pa je turšica na tablo napisala SHAKESPEARE.«(B20)

c) Le discours indirect, divisé en trois sous-catégories

Exemples:

- Le vrai discours indirect : Sem rekla Boštjanu, naj gre spat, $\mathrm{k}$ nima smisla /.../. (B1)

- Le discours indirect avec ellipse partielle du marqueur jonctif : Pol so me, ta L. me je prepričvala, ful morm it, pa obe sestri od. N., da morm it. (B12)

- Le discours indirect avec réalisation incomplète de la phrase introductive : Ta čas se vmes ni nič dogajal, tko da mene so še poslal hodit okrog, da naj se še mal sprehajam, pa to. (B1)

Chacune des catégories et sous-catégories mentionnées possède ses propres caractéristiques formelles et fonctionnelles ou métatextuelles. Il convient maintenant de les étudier plus en détails.

\section{LE DISCOURS RAPPORTÉ DIRECT AVEC « ELLIPSE PARTIELLE » DU VERBE D'ÉNONCIATION/D'OPINON DANS LA PHRASE INTRODUCTIVE}

Dans la langue parlée, il est fréquent de rencontrer une forme de discours direct caractérisée par une phrase introductive où le verbe d'énonciation ou d'opinion n'est 
pas complètement réalisé ou bien n'est pas remplacé par un autre moyen linguistique équivalent sur le plan sémantico-fonctionnel. Dans la phrase introductive, seuls sont exprimées l'identité du locuteur de l'acte de parole primaire et la forme verbale conjuguée de l'auxiliaire être.

\section{Exemples :}

- K sta pršla unadva, sem rekla: » O šit, zdaj pa...« Tolk, da sta čakala..., do polnoči sta bla pri nama, $\{e e e\}$ ob ene osmih zvečer... ampak vseen, jaz sem: "Daj bodta še mal $\{H\}$, mene je mal strah, a veš«. (B1)

- In $\{e\}$ tko ful enga folka in skratka da bi šli na ta žur, tud mi, in tko mislim, tud jaz pa L. pa to, in jaz sem najprej: »Joj, jaz ne bi šel, « pa ful sem travmiral pa to. (B12)

Dans les deux cas, nous pouvons remarquer dans la phrase introductive une sorte d'autonomisation de l'auxiliaire biti (« être »). ${ }^{1}$ Pour définir cette autonomisation fonctionnelle du point de vue métatextuel, nous pouvons dire que le rôle du participe " omis » est assumé par la citation rapportant l'acte de parole primaire. Ainsi, les marques de l'acte de citation ou de transmission de l'acte de parole primaire se trouvent estompées ; le monde textuel et, de ce fait, les conditions initiales accompagnant l'acte de parole primaire sont placés au premier plan et semblent en partie s'amalgamer avec les circonstances de l'acte de parole actuel. La distance temporelle séparant les actes de parole primaire et secondaire. La citation prend donc en charge un rôle sémantique et structurel proche de celui assumé par le participe dans la formation de la forme verbale du passé. Ce que nous venons d'écrire signifie que, lorsque nous avons affaire à ce type de discours rapporté, nous ne devrions pas parler d'ellipse du participe passé, puisque la forme est en réalité pleinement réalisée sur les plans sémantique et structurel. Dans ces cas, l'emploi du participe serait redondant et l'assimilation de cette absence à une omission (ellipse) du participe est un non-sens, vu que le co-texte, les circonstances extérieures et les structures syntaxiques potentielles n'appuient pas cette interprétation. Nous pouvons donc parler d'ellipse exclusivement en se basant sur une comparaison structurelle (sur la prise en compte d'écarts structurels) entre la formation habituelle du discours direct dans la langue standard et la formation de ce même discours direct dans la langue parlée.

- Rekla mi je petnajsti do dvajseti oktober, ne, no pol sem pa jaz enaindvajsetega rodila, ampak je blo, $\{e m\}$, že tolk, razumeš, da me je tast na hodniku, $k$ sva se srečala, ne: "Ja a si še kar doma? Ej, pismu, mislim, k bi lohk saj te, k so bli domači ne težil. (B1)

\footnotetext{
${ }^{1}$ Quand la phrase est au passé, en principe la phrase introductive est formée du pronom personnel (qui est le plus souvent ommis), de l'auxiliaire être et du participe (par exemple : (jaz) sem rekel « j'ai dit »). Dans les échanges oraux spontanés, nous avons affaire à une structure différente où le participe est ommis. Ainsi, le locuteur ne réalise que le pronom personnel et l'auxiliaire être.
} 
Dans ce dernier exemple, la locutrice exprime dans la phrase introductive l'identité de l'auteur de l'acte de parole primaire (tast - «mon beau-père »), l'identité du récepteur de l'acte de parole primaire (me - accusatif du pronom personnel « je ») et les circonstances spatio-temporelles dans lesquelles l'acte de parole primaire a eu lieu ( $\mathrm{na}$ hodniku, ko sva se srečala - " dans le couloir, quand nous nous sommes rencontrés »). Le pronom personnel exprimant le récepteur de l'acte de parole primaire est à l'accusatif. Lorsque la citation assume le rôle de complément sémantico-structurel de l'auxiliaire biti - être (ici : je - « est »), l'accusatif est le cas le plus fréquemment employé pour désigner le récepteur de l'acte de parole primaire.

\section{LE DISCOURS RAPPORTÉ DIRECT AVEC « ELLIPSE TOTALE » DU VERBE D'ÉNONCIATION/D'OPINON DANS LA PHRASE INTRODUCTIVE}

Il convient de présenter une forme plus fréquente que celle évoquée précédemment : la forme où la phrase introductrice ne comprend aucune forme verbale - conjuguée ou non - ayant pour fonction d'annoncer la citation.

Exemples :

- A: Virus je dobila in je bla v bistvu ene štiri dni tam s takim (G: kaže s kakšnim) nosom, ne. No, medve sva čaj, $\{e m\}$ tist čaj natepavale, ona je tisto tortico, pol je pa rekla: »Zdaj pa moram it « «e, »v Ljubljani moram bit ob dvanajstih, « k ma pač zamenjavo, ne, ker ma prvi razred devetletke in unadva: »Midva bi šla pa s tabo v Celovc. A lahko greva?» ne. K je še tolk ur blo, veš, to je blo pet ur za čakat. Midve sva ble..., ob devetih smo bli že tam, a veš, ob dveh pa trideset je bil pa let, no, še več, ne.

M: $\{$ Uuu. $\}$

A: Ja, no in, pol unadva: »Ja, kdo bo pa naše kufre čuvu, ne?»

$\mathrm{M}:\{H\}$

A: A veš, in pol jaz nisem hotla nič rečt, ne, in, in potem je Sandra že šla. Je rekla: „Kaj je zdaj? Vidva gresta z mano do Celovca al ne? « In pol midve tko tih, ne, a veš, jaz tko izdajalska do svojga bratranca, ne, zamera pa to, in pol Mateja tko, veš, čist na koncu, k..., veš, Sandra je bla že tam zunaj, ne, ker je mela tud sam pol ure parkirišča, ne, in pol ona tko milostno, merciful: "Lahko gresta, okej bova pogledale, sam dol jih pa ne bova..." a veš, ker smo bli gor na galeriji, v kafiču, je rekla: »Dol jih pa ne bova nosile, če ne bosta pršla do takrat.«, ne. In pol una: »Hvala, hvala,« in sta šla.

M: Ja. (B5)

- Pol sva začela si o čustvih govort, jaz tko njej:»Joj, N., jaz te ljubim, $\{e\}$ nikol v živlenju nisem bol ljubil..."

S: A ti to resno, al kaj?

K: Ja ja ja, $\{a h a\}$, tko po telefonu, in ona: »Ja, jaz tud tebe ljubim, « in tko sva si totalno ljubezen izpovedala prek telefona. (B12)

Dans le première exemple, nous pouvons constater une structure intermédiaire entre, d'une part, le discours rapporté avec phrase introductrice où le rôle d'annonce de la citation est assumé non seulement par le pronom personnel, mais aussi par la forme verbale conjuguée (je rekla) et, d'autre part, le discours rapporté avec phrase introductive où seul 
le pronom personnel mentionnant l'auteur de l'acte de parole primaire (unadva, ona) est exprimé. Il est intéressant de constater que la locutrice à « omis » la forme verbale conjuguée dans les phrases introductives annonçant des événements/citations particulièrement importants, étonnants, inattendus ou captivants. En « omettant » le verbe d'énonciation/d'opinion ainsi que les précisions concernant les autres circonstle verbe pravitiances, la locutrice oriente l'attention du récepteur directement et exclusivement sur l'acte de parole primaire. Ce faisant, elle le souligne et le met en valeur. Avec l'omission de la forme verbale, la dimension temporelle de l'acte de parole primaire (le passé) reste également inexprimée, ce qui a pour effet d'associer en apparence l'acte de parole primaire à la dimension temporelle de l'acte de parole secondaire (le présent).

- In pol spet s to kartico, ne. Jaz sem šla kar stran, veš, res nisem mogla bit tam zraven, ne. In ona men tko čez cel oddelk otroških igrač: »Spet je ratal.« (B5)

Dans ce dernier exemple de discours direct avec « ellipse totale » du verbe d'énonciation ou d'opinion dans la phrase introductive, la locutrice mentionne (seulement) l'auteur de l'acte de parole primaire (ona), le récepteur (men) et le lieu où l'événement narré a eu lieu (čez cel oddelk otroških igrač). Dans les phrases introductives avec « ellipse totale » du verbe d'énonciation/d'opinion, on rencontre toujours, en règle générale, un lexème assumant le rôle de sujet (l'auteur de l'acte de parole primaire) sous la forme d'une particule de phrase. Cela n'a rien d'étonnant, car il s'agit là du seul moyen disponible (en dehors des moyens anaphoriques possibles) permettant de se référer à l'auteur de l'acte de parole primaire.

\section{LE DISCOURS RAPPORTÉ DIRECT SANS PHRASE INTRODUCTIVE}

Le rôle métatextuel consistant à rapprocher le monde textuel du récepteur et à accroître progressivement l'attention du récepteur en renforçant le rôle conatif est assumé également par les formes de discours rapporté où les phrases introductives semblent avoir été omises.

Exemples:

- P: Jaz niti dihat nisem znala. Matjaž prav: »Dihat dihat.« Pride sestra notr: »A ste hodila na šolo za starše?" »Sem. « "Ja, pa saj so vas učil tam dihat." »So, ampak men ne gre. Dejva skupi.." No, pol sva skupi dihale, mislm, po pa, $k$ je šla ona ven, pa jaz tko: »Matjaž, jaz to škodo delam dujenčku. Jaz sploh ne diham prou, sploh ne diham v trebuh, jaz kar nekam diham, sam da diham.«Prav: »Ne ne, saj bo, saj bo.« Pol je šu mal za hrbet mi, pa me je mal masiru, /.../ in pol sem spala in spet, ne. Ampak to, ne, in pol kar naenkrat so pršle: »Kera ekipa, \{aaa\} gospa, zdaj pa na ta drug bok, pa noge skup pa fajn prtisnte, " pa smo trikrat tlele, "pa nogo pa glavo naprej, pa še na unga." Pa me je umes ubi- uuujel pupadek, k sem glih na hrbet, pa, a ne, k še nisem do naslednga boka pršla. (B16)

- M1: Bla sem tam, sem se kar sprehajala ene dve uri. Pa pridem nazaj, pa spet una pride: "Ja, nič. « Pupadki so pa minil, mislim minil, niso bli več tko na pet minut, ampak kar na deset pa, taki švoh, ne, mislim tko. 
M2: $\{$ Mhm $m h m$.

M1: Pol je, pol je ob pol devetih je bil Boštjan že tam. Pride en, a je to stažist, al kaj jaz vem kaj, en mlad dohtar, $k$ še ni niti ni dohtar, mislim, da je stažist, ne, pa tud če ne, no: "Ja, bomo kar mehur prepiknil, ne." (B1)

Dans les deux cas, nous voyons clairement que, fréquemment, les citations ne sont précédées d'aucune phrase introductive classique annonçant 1 a mention des actes de parole primaires. Dans le premier exemple, nous rencontrons au début la reproduction littérale du discours primaire sans aucune phrase introductive (Pride sestra notr. "A ste hodila na šolo za starše?" "Sem." "Ja, pa saj so vas učil tam dihat." "So, ampak men ne gre. Dejva skupi.«). ${ }^{2}$ Un examen plus attentif de ce cas montre que, en réalité, l'ellipse des phrases introductives n'est qu'apparente. L'annonce de la citation est le plus souvent prise en charge par le verbe priti au passé ou au présent (présent historique). Ce dernier voit son champs sémantique élargi et remplit au moins deux fonctions à la fois : il recouvre son champs sémantique primaire et, par élargissement sémantique, accroit la tension dramatique de l'acte de parole primaire ou de l'événement passé dans lequel s'inscrit l'acte de parole primaire.

\section{LES DEUX VERBES SIGNIFIANT « DIRE », REČI ET PRAVITI, DANS LA PHRASE INTRODUCTIVE}

L'un des verbes d'énonciation les plus fréquents dans les phrases introductives ou introductives est le verbe reči (dire). Par ailleurs, les locuteurs emploient souvent également le verbe praviti (dire). Bien que leur usage soit habituel, il est intéressant d'analyser leurs formes et les cas où ils sont en concurrence.

Exemples:

- Prideva tja. Najprej tam mi CT delajo, pol me pregleda, prav: "Tri pa pol samo odprt.« Sem rekla: „Pol grem lahka dam.« Prav: »Ne, lahko greste gor v sobo al pa v porodno.« (B1)

- K je bil Val majčkn, /.../ in jaz mu \{ee\} kupim en Kinder jajček, je že takrat norel za temi Kinder jajčki, ne, in on je spet to reku Kindel solplajs, in $\{e e\}$ je skratka ... sedi na kahlci pa prav: »Dida, odvi mi, « ne, jaz pa v tisti naglc, mislim, da sem hotu prpravt mu tist njegov zrezek, ne, k je stalno meso jedu, ne, \{ee\} rečem: »Joj, Val, ti bom pol, ne, rajši, ne. Zdele nimam časa. « \{ee\} Pa prav: »Ne, kar odvi, « in sem mogu odvit, ne, potem je pa reku: »Ja," čez neki časa, seveda tam notr je tist jajček, ne, $\{e e\}$ čukuladnega itak on ni nikol pojedu, ga je kar zraven odložu, pa tko naprej, ne, nakar je \{ee\} tist jajček..., je reku: »Ja, pa tud $u d r p r i$, ne.« No, po sem pa še tist, sem se udal v usodo, ne, sem reku, zdaj bo dal mir, k bo vidu, kaj je notr, kakšna stvar, ne, kaj za sestavt, al, ne vem, ne, in skratka, je po tem [ee] no, $\{e e]$ grem jaz nazaj s h tisti svoji kuhi, ne, $\{e e\}$ nakar on prav: »Dida, $\{e e\}$ na zdaj mi pa puvej, kako se to sestav, bova sestavla, ne.« Jaz sem reku: »Val, veš kaj, nimam špeglou tuki, ne.« Potem se pa zlo zamisl, pa neki časa je tih, potem pa prav, ko gleda tist listek,

${ }^{2}$ Trad. « L'infirmière est entrée. 'Vous avez suivi le stage pour les futurs parents ?' 'Oui.' 'Eh bien, là-bas, ils ont bien dû vous apprendre à respirer, non ?' 'Oui, mais moi je n'y arrive pas. Allons-y ensemble.' » 
ne, na kterem je blo pač shema za sestavt tale $\{e e\}$ tisto igrco v Kinder jajčku oziroma tist tist predmetek, ne, in prav: "Dida, tuki piše Ne jabiš očal.» (B30)

- Nobenga človeka nisva srečala, no, pol prav Nastja ..., jaz sem šel glih lu- lulat, /.../ sem reku: "Ti pejt pa mal naprej, pa poglej, če tam glih približn pri križu, če kakšen dol pride, " pa prav: "Dva gresta, dva gresta.« Jaz hiter pol naprej, pol pa vidm..., enga znanca vidm, /.../, [ee \} hudiča, ves tak je bil, tkole $\{H]$ ves pobit prav, pa smo se tem ene deset minut pogovarjal pa to, pa prav Nastja: "Tale ga pa fejst pije." Sem reku: »Ja, prav, zakuga?« »Ja, zato, saj svidu, kakšen je." »A misliš, da zato, k je pobit? «»Ne, "prav »zato k ga v ksiht pogledaš. «/.../ No, pol midva sva hotla it tam naprej še potem do do koče $\mathrm{v}$ Vojah, $\{e e\}$ no, pol sta pa unadva rekla..., sta šla pa dol, pa $\{e e\}$ ob koritih gor nazaj, ne, pol sem reku: "Tle je zdaj nevarnost, da se že spet srečamo, pa debatirat, na konc se bomo še domu povabil al kaj tazga, pejva midva pol tam." (B11)

Dans les trois exemples, l'analyse des verbes reči et praviti montre que l'emploi du verbe praviti est avant tout lié à l'interlocuteur de l'acte de parole primaire et apparaît à la $3^{\mathrm{e}}$ personne (on/ona prav-i - «il/elle dit »). Dans tous les exemples retranscrits précédemment, le verbe praviti est toujours employé au présent (présent historique) et à la $3^{\text {e }}$ personne. En revanche, le verbe reči est avant tout lié à la première personne (sem reku/rekla - «j'ai dit»), donc au locuteur qui rapporte l'acte de parole primaire. Contrairement au verbe praviti, il est le plus souvent employé au passé. Du point de vue métatextuel, cet emploi est habituel. Par son aspect imperfectif et son présent, le verbe praviti introduit une tension, fait croître l'attention du récepteur. Du point de vue du locuteur et de son appréhension du monde textuel et de l'événement narré lui-même, les déclarations de l'interlocuteur de la situation de parole primaire représentent une péripétie, un tournant inattendu. Ainsi, la tension dramatique ou péripétie caractérisant la citation des interlocuteurs de l'acte de parole primaire est le plus souvent soutenue précisément par un choix lexical (le verbe praviti opposé à reči) et la forme verbale elle-même (présent historique/véritable présent, aspect perfectif/imperfectif).

Les déclarations du locuteur de l'acte de parole primaire, qui est en même temps le rapporteur de ce dernier, ne constituent pas pour le locuteur une péripétie, un tournant illogique, c'est pourquoi ce dernier annonce le plus souvent ses autocitations à l'aide du verbe reči au passé.

\section{LE DISCOURS INDIRECT AVEC RÉALISATION INCOMPLÈTE DE LA PHRASE INTRODUCTIVE}

La langue parlée se caractérise aussi par le fait que le discours rapporté y est cité à l'intérieur d'une phrase introduite par une conjonction, mais dont la réalisation est interrompue, incomplète. Ici, le discours rapporté est toujours introduit par une conjonction de subordination (par exemple, $d a$ - « que », če - "si ») qui, précisément du fait que la phrase introductive reste non réalisée, assume un rôle élargi.

Exemples :

- Pol je, pol je ob pol devetih je bil Boštjan že tam. Pride en, a je to stažist, al kaj jaz vem kaj, /.../ »Ja bomo kar mehur prepiknil, ne.« Pa gre z uno, tako iglo (G: kaže z rokami, 
kako dolga) noter, veš. /.../ Je blo pa z vodo čist vse v redu, ne, $\{$ em $\}$, ja, da bo to pospešil, ne. Pol pa, $\{e m\}$, ni blo nič bolš, pol sem mela pa še umetne. (B1)

- Me pokliče N. »[Neee]«, če gremo ven, in da bo tud L., to je ta njena najboljša prjatelca, $\mathrm{v}$ bistvu smo bli skupi v Rimu, trojka, v bistvu, in smo bli tam en teden, cel žur in to, skratka, tko, dokaj prjatli, pa to. No in $\{e\}$, me skratka pokliče in $\{e\} \check{c} e$ gremo ven, tko bla bla bla in sem reku: »Ja valda, the best.« (B12)

- Sem mela enga, k je reku, da je Jezus Kristus. Pršu je, pa niti ne moj pacient, sem bla dežurna, in da ga boli, /.../, mulc, sem rekla: »Tle je treba sam trakec dat notr, pa pač menat, saj bo nehal tekom enga tedna, « ne. In drug dan je pršu, da nega tok boli, je reku..., pa že prej sem vidla, da ni pr č- čisti, k je začel, če ga bo nehal, da pol mu verjetn ni treba it samomora delat, zarat tega, ne, pa take fore. No poj pa drug dan pršu, da ga še zmer boli, sem rekla: »Ja, v redu.«(B25)

Si nous observons de plus près le premier et le troisième exemples, nous constatons que la conjonction $d a$ (« que ») introduisant l'acte de parole primaire y revêt un rôle élargi. En dehors du rôle consistant à introduire, la conjonction da endosse également un rôle proche de celui d'une particule modale. En effet, le locuteur l'utilise pour exprimer son point de vue subjectif et ainsi commenter la déclaration/citation de l'interlocuteur du premier acte de parole (par exemple, en exprimant un doute concernant la véracité d'une affirmation). Si nous examinons le deuxième exemple, nous pouvons conclure que, ici aussi, le rôle subordonnant initial de la conjonction če (" si ») est élargi. La seule différence réside dans le fait que, dans ce cas, ce rôle n'est pas modal (il ne consiste pas à exprimer un point de vue subjectif), mais consiste à annoncer le résumé (et non la citation intégrale) de l'acte de parole primaire.

\section{CONCLUSION}

D'après Slovenska slovnica (2000 : 653-656), nous pouvons identifier dans la langue parlée différentes formes de discours rapporté absentes de la langue écrite standard. Après analyse, nous constatons que les formes de discours rapporté étudiées se rencontrent essentiellement dans la langue parlée en raison de différents facteurs conditionnant la formation du texte parlé (la prosodie, la simultanéité de l'énonciation et de la réception du texte, les signes de communication non verbale venant du locuteur, diverses lois syntaxiques et textuelles propres à la langue parlée, etc.). En dehors des caractéristiques spécifiques au niveau structurel ou formel, toutes les catégories de discours rapporté observées présentent des particularités également au niveau métatextuel (tentative de rapprocher le monde textuel du récepteur, association des dimensions temporelles des actes de paroles primaire et secondaire, gradation du rôle conatif, gradation du caractère dramatique de l'événement raconté, etc.).

Il paraît évident que l'observation des emplois et des différentes formes du discours rapporté est intéressante et nécessaire non seulement dans la perspective d'une comparaison entre les langues standard et parlée, mais aussi dans le but d'établir une analyse contrastive du slovène et des autres langues. En effet, s'il est courant en slovène 
d'omettre la phrase introductive ou de substituer le verbe d'énonciation par un verbe d'opinion ou par un mot une autre catégorie grammaticale, il n'en est pas de même, par exemple, en français où cet usage est connoté, voire impossible. Formons des vœux pour que le présent article encourage de futures analyses contrastives ayant pour sujet d'étude le discours rapporté dans différentes langues.

\section{Bibliographie}

ČERnE, Danica (2012) « (Ne)prevedljivost pogovornega jezika v delih Johna Steinbecka. » Slavistična revija 60/2, 131-149.

KoroŠEC, Tomo (1998) Stilistika slovenskega poročevalstva. Ljubljana: Kmečki glas. KRIŽAJ-ORTAR, Martina (1997) Poročani govor v slovenščini. Ljubljana : Université de Ljubljana.

RosiER, Laurence (2008) Le discours rapporté en français. Paris : Ophrys.

SMOLEJ, Mojca (2006) Vpliv besedilne vrste na uresničitev skladenjskih struktur (primer narativnih besedil $v$ vsakdanjem spontanem govoru). Ljubljana : Université de Ljubljana.

TOPORIŠIČ, Jože (2001) Slovenska slovnica. Maribor: Obzorja.

\section{Résumé \\ LES SPÉCIFICITÉS DU DISCOURS INDIRECT DANS L'ÉCHANGE ORAL SPONTANÉ EN SLOVÈNE}

Contrairement à ce que l'on peut constater dans d'autres langues (notamment en français), le discours indirect en slovène est caractérisé par la possibilité laissée à l'énonciateur de faire l'ellipse du verbe d'énonciation pour lui préférer un verbe exprimant son opinion ou son état d'esprit. Or, si on aboutit dans la langue écrite standard à une ellipse partielle (par exemple, le verbe d'énonciation est remplacé par un verbe d'opinion dans la phrase introductive du discours indirect), en revanche on peut souvent remarquer dans les énoncés tirés de la langue parlée spontanée une ellipse totale (la phrase introductive ne comporte aucun verbe d'énonciation, d'opinion ou d'état d'esprit). Dans bien des cas, les phrases introductives n'indiquent que l'auteur de l'acte discursif initial et le destinataire de ce dernier (singulièrement mentionné le plus souvent à l'aide de l'accusatif). En dehors de l'ellipse complète du verbe dans les phrases introductives, on distingue également d'autres types de transmission du discours indirect absentes des autres formes écrites ou orales de la langue. Parmi les types de discours indirect les plus fréquents, mentionnons le cas où seule la citation est fournie, introduite par un signal discursif accompagné du lexème « da » (« que »), tandis que la phrase principale ou introductive reste non réalisée. En tant que structures caractéristiques du discours parlé spontané, les formes de discours indirect mentionnées précédemment présentent un intérêt non seulement pour les grammairiens étudiant la syntaxe structurale, mais aussi, plus généralement, du point de vue discursif et métatextuel.

Mots-clés : discours indirect, types de discours indirect, langue parlée, phrases introductives, ellipse du verbe. 


\section{Povzetek \\ POSEBNOSTI POROČANEGA GOVORA V SPONTANO GOVORJENIH BESEDILIH V SLOVENŠČINI}

Za posredovanje poročanega govora $v$ slovenskem knjižnem jeziku je značilno, da je v nasprotju z nekaterimi drugimi jeziki lahko glagol rekanja eliptično izpuščen, uporabljen pa glagol, ki razkriva mišljenje ali duševno stanje. Če je za knjižni jezik značilna delna elipsa (v spremnem stavku je npr. uporabljen glagol mišljenja, ne pa glagol rekanja), je v spontano govorjenih besedilih velikokrat zaslediti polno elipso (v spremnem stavku ni izraženega glagola rekanja oz. mišljenja ali duševnega stanja). V spremnih stavkih v spontano govorjenih besedilih sta tako nemalokrat podana le tvorec prvotnega govornega dejanja in naslovnik prvotnega govornega dejanja, ki pa je presenetljivo največkrat izražen v tožilniški obliki. Poleg polne elipse glagola $\mathrm{v}$ spremnih stavkih je $\mathrm{v}$ spontano govorjenih besedilih zaznati tudi druge vrste posredovanja poročanega govora, ki kot take niso pričakovane v drugih jezikovnih zvrsteh. Pogoste so oblike poročanega govora, kjer je podan le citat, ki ga uvaja določen govorni signal skupaj z leksemom da, spremni oz. glavni stavek pa ostaja neizpeljan. Omenjene oblike poročanega govora v spontano govorjenih besedilih niso zanimive le s stališča slovničnega oz. strukturnoskladenjskega raziskovanja, pač pa predvsem s širšega diskurznega in metabesedilnega pristopa.

Ključne besede: poročani govor, oblike poročanega govora, govorjeni jezik, spremni stavki, elipsa glagola. 